Cécile FER

Université Palacký d'Olomouc

\title{
L'EXTRA-LINGUISTIQUE DANS LES PROCEDES DE TRADUCTION : ANALYSE DE QUELQUES EXEMPLES ISSUS DE LA COMPARAISON DES TRADUCTIONS FRANÇAISES DE LA PLAISANTERIE DE MILAN KUNDERA
}

\section{Introduction}

Le processus de traduction n'est pas simplement la confrontation de deux langues face à une réalité qui serait identique, mais aussi la confrontation de deux réalités : il s'agit effectivement par la traduction de passer d'une langue à l'autre et également d'un univers extra-linguistique à l'autre, d'une expérience collective cognitive à une autre, pour reprendre les mots de M. Srpová ${ }^{1}$.

Georges Mounin, dans Les Problèmes théoriques de la traduction ${ }^{2}$ le dit aussi à sa façon: "Prétendre - puisque chaque langue est décrite comme un système algébrique de relations et de corrélations formelles - que la traduction peut être réduite à des problèmes de conversions algébriques formelles, au passage mécanique des formules linguistiques d'un système (le russe, par exemple), aux formules linguistiques d'un autre système (le français), c'est une vue partielle qu'on prend pour une vue totale .» Pour lui, si la traduction "qui est une série d'opérations dont les matériaux initiaux et finaux sont des significations [...] peut avoir recours à une linguistique formelle », elle doit finalement «assigner des valeurs sémantiques concrètes aux formules vides: rentrer dans le monde des significations. ${ }^{3} \gg$ On comprend alors le titre qu'il a donné au chapitre en question : «l'ethnographie est une traduction ».

C'est précisément ce qui est du domaine extra-linguistique et qui touche donc à l'ethnographie, qu'il est intéressant d'analyser à travers la première traduction de La Plaisanterie de Milan Kundera faite par Marcel Aymonin en 1968, et la version définitive revue par l'auteur et Claude Courtot en 1985.

Il faut ici rappeler que s'il est un écrivain tourmenté par le processus de traduction, c'est bien M. Kundera, dont l'écriture elle-même prend parfois pour thème les écarts entre l'expérience tchèque et l'expérience française : il a par exemple failli intituler L'insoutenable légèreté de l'être, «La planète de l'inexpérience », et il consacre la sixième partie de L'Art du roman « Soixante et onze mots », à une sorte de dictionnaire des mots incompris.

L'analyse de plusieurs exemples de modifications apportées par M. Kundera au travail de M. Aymonin ${ }^{4}$, permet de distinguer trois types de comportement du

\footnotetext{
1 SRPOVA Milena (1995), «La traduction, confrontation de deux expériences cognitives », Intellectica, 1, 20, p.157-170.

${ }^{2}$ Mounin Georges (1963), Les problèmes théoriques de la traduction, Paris, Gallimard, p.227.

${ }^{3}$ idem, p. 232 .

${ }^{4}$ Cette présentation contient des extraits :

-du texte tchèque qui sera désigné par l'abréviation «TCH»: Žert, édition originale 1967 (exemplaire utilisé : Brno, Atlantis, 1996).

-de sa première traduction en français par Marcel Aymonin, «TA»: La Plaisanterie, Paris,Gallimard, 1968.
} 
romancier face aux problèmes de traduction des spécificités référentielles tchèques : tantôt il a supprimé celles-ci, tantôt il les a neutralisées en les adaptant à l'univers français, tantôt il les a conservées tout en les expliquant.

\section{Neutralisation des spécificités tchèques par le procédé de suppression}

\section{1) Le traitement des noms de lieu}

TCH p.29: «[...] sehnal bůhvíjak nějakou chatu v Krušných horách »

TA p.86: " Dieu sait comment il dégota un petit chalet au fond des Monts Métalliques »

TR p.132: " Dieu sait comment il dénicha un petit chalet au fond des montagnes. »

Le dictionnaire tchèque-français de Václav Vlasák et Stanislas Lyer donne comme traduction à « Krušné hory », «monts métallifères ». M. Aymonin a donc opté pour la traduction littérale. M. Kundera a préféré neutraliser en exprimant le sens commun à la spécificité tchèque et française, en utilisant la classe, ici «montagne ».

TCH p.29: «a potom jsme sedéli v malé hospídce u Zbraslavi »

TA p.29: «et puis après, installés dans une petite auberge non loin de Zbraslav » TR p.43 : « et puis après, installés dans un petit bistrot $\varnothing$ »

Dans cet exemple, alors que chez M. Aymonin le nom de la ville entre tel quel dans le texte français, Kundera, jugeant certainement que les lecteurs français ne la connaîtront pas et que cette information ne leur apporterait rien, préfère ne pas préciser le lieu de l'action.

TCH p.139: «Ludvik odjel studovat do Prahy, já do Brna. Oba jsme nechali doma dva opuštěné lidi. Ludvík maminku, já tatínka. Brno je naštěstí od nás dvě hodiny vlakem. Ale Ludvíka jsem po maturitě celý rok nevidèl. »

TA p.159-160 : "Ludvik s'en fut étudier à Prague. Moi-même à Brno. Nous laissions derrière nous deux êtres à l'abandon. Ludvik sa maman, moi, papa. Heureusement, Brno se trouve à deux heures de train de chez nous. Ca me permettait de rentrer pour chaque week-end. Retrouver papa et l'orchestre. Cependant, après le bachot, je ne revis pas Ludvik de toute l'année. »

TR p.210: "[...] Ludvik s'en fut étudier à Prague, moi à Brno. Je ne le revis pas de toute l'année. "

Ici la traduction de $\mathrm{M}$. Aymonin va plus loin que le texte tchèque : celui-ci semble juger nécessaire d'expliquer aux lecteurs français ce qu'implique la phrase «heureusement, Brno se trouve à deux heures de train de chez nous»: le personnage va pouvoir rentrer dans sa famille le week-end. Cette fois-ci M. Kundera ne supprime pas les localisations puisqu'il s'agit des deux villes tchèques les plus importantes, en revanche il n'indique pas, comme dans le texte tchèque, la

-de la même traduction française révisée par Claude Courtot et l'auteur, "TR », dont la version définitive est parue dans la collection de poche Folio des éditions Gallimard en 1985 (il y avait déjà eu une première révision de traduction éditée en 1980). 
distance, et abandonne l'idée de M. Aymonin de décrire l'habitude des étudiants tchèques, qui consiste à faire facilement un trajet de deux heures de train pour rentrer chez eux le week-end, à préférer le foyer familial aux cités universitaires peu confortables. Outre la précision géographique des distances, c'est donc un aspect sociologique, à savoir le quotidien d'un étudiant tchèque, qui est supprimé ici par rapport au texte tchèque et à la traduction de $\mathrm{M}$. Aymonin.

\section{2) Le parti pris de la suppression de certains traits culturels propres à la République tchèque}

Révisant la traduction de M. Aymonin, confronté par exemple à la traduction d'une description de façade d'un bâtiment, et à celle d'une coutume appartenant au folklore et aux fêtes traditionnelles, M. Kundera a opté pour la suppression pure et simple de paragraphes entiers du texte tchèque.

TA p.190 (cf. TCH p.168) : «Il s'y dresse une massive construction d'un étage flanquée de deux espèces d'échauguettes sur les versants du toit; dans la façade s'encastrent, au niveau du rez-de-chaussée, quatre baies-vitrines avec, au-dessus de chacune, une lunette; sur la vitre de la première on a figuré au pinceau trois bonhommes en costume national, mains enlacées sur les épaules et bouche ouverte; sur la suivante, un couple (également en costume) ; sur la troisième, un tournesol et un tonnelet de vin sur la dernière. C'était un libre-service. »

Peut-être M. Kundera a-t-il jugé dans ce cas précis qu'une lecture profitable de cette description supposait une connaissance préalable des traits généraux de l'architecture en République tchèque, sans laquelle la visualisation du bâtiment demanderait un effort trop grand au lecteur et n'apporterait rien au sens global du texte.

TA p.98-99 (cf. TCH p.88) : "J'étais un vrai veau à l'époque, je me rappelle qu'elle adorait d'être battue sur ces fesses-là et moi qui ne pigeais toujours pas! Une fois, c'était au moment de Pâques, fallait surtout pas que j'oublie d'amener des baguettes tressées*, bon, des salades à n'en plus finir, et quand j'ai été là, alors, tape, qu'elle disait, tape sur la petite dame, vas-y, n'aie pas peur, t'auras un ouf peint; moi, je cognais mou, sur la jupe, et elle râlait: c'est ça que t'appelles battre? Allez, relève-lui sa jupe, à la petite bourgeoise, bien fallu que j'obtempère et puis que j'abaisse sa culotte et moi, connard, je continuais à faire semblant de cravacher, et elle devenait mauvaise, si vous l'aviez entendue gueuler: tu vas cingler pour de bon, crevure! Bref, j'étais un pauvre con... »

*Allusion à une coutume du folklore tchèque : le matin de Pâques, les garçons, armés de verges de saule tressées et enrubannées, donnent la chasse aux belles, lesquelles, en retour, les gratifient d'eufs artistement peints.

M. Aymonin opte, afin d'expliquer la coutume, pour le procédé de la note de bas de page. M. Kundera, quant à lui, révise le texte en pensant certainement que le lecteur ne pourra rire du personnage, que s'il connaît d'avance la coutume à laquelle celui-ci fait référence. Il préfère supprimer cette référence plutôt que de tenter de provoquer l'amusement du lecteur de manière décalée dans le temps, grâce à une explication en note de bas de page dont la lecture serait sûrement postérieure à celle du passage. 
D'autres exemples montrent que M. Kundera s'est attaché à gommer, toujours par le procédé de la suppression, ce qui touchait aux modes de vie de la population pendant des périodes historiques données.

TCH p.60: "protože v bývalém sále sokolovny, na niž se žádný zákaz nevztahoval, byla tancovačka.»

TA p.64 : "il y avait une soirée dansante dans la salle désaffectée de la maison des Sokols*. »

*Sokol (Faucon) : puissante organisation d'éducation physique et patriotique fondée en 1862 et qui joua un rôle considérable dans le mouvement national d'émancipation des Tchèques.

TR p. 92: "il y avait une soirée dansante dans la salle désaffectée d'un gymnase. "

M. Kundera a traduit par le sens commun à la culture de départ, tchèque, et à la culture d'arrivée, française, en gardant la classe, ici le type de lieu, un gymnase.

TCH p.176: «Byla oblečena do modrého šustáku, italského pláště, který se v té době kupoval v Tuzexu a propưjčoval všem svým nositelkám mladistvě sportovní podobu. I Helena (měla vyhrnutý límec a přepásaný pás) $v$ něm vypadala výborně. »

TA p.201 : «Elle portait un imperméable bleu ardoise, un de ces manteaux de fabrication italienne qu'à l'époque on ne trouvait qu'au magasin Tuzex* et qui conféraient à toutes les femmes une allure jeune et sportive. Hélène aussi (son col relevé, la taille bien sanglée sous une ceinture) avait grand air dans ce trench. » *firme d'Etat spécialisée dans la vente des articles de luxe, importés ou destinés à l'exportation, payés en devises étrangères.

TR p.162: «Elle portait un imperméable bleu qui (le col relevé, la taille bien sanglée sous une ceinture) lui conférait une allure jeune et sportive. »

Comment interpréter ces suppressions, sinon en imaginant que dans l'esprit de M. Kundera, de tels détails ne peuvent avoir de sens pour le lecteur, que s'il les a luimême vécus, où s'ils font partie de son histoire. Le lecteur français, privé de ces détails, doit en quelque sorte les reconstruire, à partir de son propre degré de connaissance par exemple du régime communiste, contexte dans lequel $\mathrm{M}$. Kundera ancre le récit dès le début du roman.

\section{Neutralisation des spécificités tchèques par le procédé de l'adaptation}

\section{1) Quelques exemples qui montrent l'accord entrent la traduction de $M$. Aymonin et la version révisée par M. Kundera}

TCH p.169: «po třech minutách popocházejícího čekání jsem si koupil [...] »

TA p.191-192: « une attente raisonnable me valut »

TR p. 250 : « après dix minutes d'attentej'obtins »

Dans cet exemple, la signification hors contexte du segment à traduire est « trois minutes ", son sens dans le contexte de la culture tchèque étant une attente de durée non marquée, dans un lieu de restauration rapide et à une époque où les conditions politiques font que l'attente dans les queues est habituelle pour les Tchécoslovaques. On peut également émettre l'hypothèse que, la culture tchèque 
se caractérisant par un éthos de vérité, la langue tchèque dira «trois minutes » si le temps réel est effectivement de trois minutes. La différence extralinguistique entre l'expérience tchèque et l'expérience française se situe ici au niveau du rapport au temps. Les Français, à l'opposé des Américains par exemple, comme le montre E.T. Hall, supportent plutôt mal l'attente dans une queue, et ils prennent la mise sur un pied d'égalité qu'elle constitue, comme une atteinte à leur individualité. Ainsi, même si le temps réel est de trois minutes et que le temps de l'attente est non marqué dans la culture française, la langue française exagèrera cependant la durée de l'attente. M. Aymonin est resté, dans sa traduction, au niveau de l'interprétation du segment à traduire dans la culture d'arrivée, à savoir une attente non marquée, qu'il exprime par « une attente raisonnable ». Kundera va plus loin en réexprimant le sens par une expression équivalente en français, « une attente de dix minutes ».

TCH p.27 : « bez kouska solidarity k stař̌i ženě, vždyt’ jim taky jednou bude třicet a pětatřicet a čtyricet. »

TR (=TA), p.39 : «dépourvues du moindre brin de solidarité avec la femme un peu plus âgée, comme si un jour elles n'auront pas à leur tour trente ans et trentecinq ans et quarante ans. »

La différence extra-linguistique touche ici le rapport à l'âge, concernant les femmes. Le texte tchèque considère comme «plus âgées », "starší », des femmes de trente, trente-cinq et quarante ans. Dans son article «Problèmes de la traduction, enjeu d'une œuvre littéraire ? $»^{5}$, M. Srpová explique qu'à l'époque où se situe l'action des romans de M. Kundera, une femme de trente-cinq ans n'est plus considérée comme jeune en Tchécoslovaquie, alors qu'elle l'est encore en France, ce qui est exprimé dans la traduction française par souci d'adaptation, par le comparatif atténué d'un adverbe, « un peu plus âgée ».

Voici un dernier exemple qui montre une adaptation d'une spécificité tchèque, le parler populaire de Honza, écart par rapport au tchèque standard. Il est réexprimé dans la culture d'arrivée, par l'emploi d'un idiolecte représentant un écart par rapport au français standard, marquant moins comme en tchèque une différence de niveau social qu'une différence régionale, puisqu'il s'agit d'une expression propre au sud de la France :

TCH p.60 : « Vole, a my sem patríme? »

TR (=TA) p.91: « Eh con ! Et nous, qu'est-ce qu'on a à faire ici ?»

\section{2) Adaptations de M. Kundera, là où M. Aymonin jouait l'effet « couleur locale »}

TCH p.21 : «jen spořádaný byt spořádaných plzeňských občanů »

TA p.19: «[...] que l'appartement bien rangé de la bourgeoisie bien rangée de Plzen $\gg$

TR p.30: «que l'appartement bien rangé de la bourgeoisie provinciale bien rangée »

\footnotetext{
${ }^{5}$ SRPOVA Milena (1988-89), « Problèmes de la traduction, enjeu d'une œuvre littéraire ? », Études tchèques et slovaques, Presses universitaires de Paris Sorbonne, ${ }^{\circ} 7$, p. 81-110.
} 
M. Kundera remplace ici le nom propre de lieu, par un adjectif qui donne une équivalence de sens dans la culture française, car il résume ce qu'il est important que le lecteur retienne de l'origine des personnages, c'est-à-dire à quelle partie de la société ils appartiennent.

TCH p.168: «morovým sloupem»

TA p.190 : «colonne de la peste*»

*Monuments de style baroque ou rococo érigés aux XVIIème et XVIIIème siècles sous l'impulsion de la Compagnie de Jésus, après les épidémies de peste.

TR p.249 : «monument baroque »

Autant l'adaptation pouvait paraître impossible pour tout ce qui concernait la période communiste, autant les «colonnes de la peste » peuvent trouver pour un Français un équivalent approximatif dans l'expression "monument baroque », étant donné qu'il en existe aussi en France, même s'ils sont de nature différente.

\section{Quelques exemples de conservation des spécificités culturelles tchèques, accompagnées d'explication}

\section{TCH p.59 : «dvacetiletý Stáňa, ztřeštěný žižkovský frajer »}

TA p.63 : "Stáňa, vingt ans, un gandin maboul de Prague-Zizkov »

TR p.90 : «Stana, vingt ans, un dandy d'une banlieue ouvrière de Prague »

La traduction littérale de $\mathrm{M}$. Aymonin, traduit l'adjectif tchèque par un syntagme nominal qui contient le nom propre «Žižkov » à l'origine de ce mot et également une explication: le fait d'ajouter au nom de départ, «Prague », pour former une sorte de mot composé qui a des airs de locution figée, permet au lecteur français de comprendre qu'il doit s'agir d'un quartier de Prague. M. Kundera abandonne une fois de plus une partie de l'effet «couleur locale» produit par le nom propre « Žižkov », pour le traduire par sa classe puisqu'il le rend par le mot «banlieue ». Il en profite aussi pour expliciter le fait que celle-ci est essentiellement ouvrière, détail qui lui semble important pour la contextualisation, et que M. Aymonin n'avait pas jugé utile d'expliquer au lecteur.

TCH p.22: «moravské pisně jsem si tehdy tak zamilovala, že jsem se cítila, já, Plzeňačka, Moravankou, staly se leitmotivem mého života. »

TA p.19: «je m'étais alors à ce point éprise des airs de Moravie que moi, native de Plzen, je me sentais morave, j'ai fait de ces chansons le leitmotiv de mon existence. $»$

TR p. 30 : «[...] moi, native de Bohême, je me sentais morave.»

Le choix de traduire Plzen par le nom de la région à laquelle cette ville appartient, permet d'expliquer au lecteur que le sentiment d'appartenance à la Moravie est d'autant plus étonnant chez ce personnage, qu'il est originaire d'une région qui se définit par opposition la Moravie, à savoir la Bohême.

Dans les deux exemples suivants, l'explication à caractère ouvertement pédagogique des traits culturels conservés, au lieu de se trouver dans le paratexte, c'est-à-dire en note de bas de page comme très souvent chez M. Aymonin, est insérée chez M. Kundera dans le texte, d'ailleurs parfois de manière plus ou moins heureuse. 
TCH p. 40 : « К̌ekl jsem "čest práci", on nepozdravil a řekl»

TA p. 38 : «Je dis "Honneur au travail"**, il ne répondit pas à mon salut et dit »

*Formule de salut quasiment "réglementaire » entre communistes tchécoslovaques, aujourd'hui à peu près abandonnée.

TR p. 58 : «Je dis "Honneur au travail "comme les communistes se saluaient à l'époque. Il ne répondit pas à mon salut et dit »

TCH p. 292 : «navrhl jsem vodku, ale odmitl ji, protože prýř́idí.»

TA p. 319: "je proposai de la vodka, qu'il refusa en faisant remarquer qu'il conduisait*.»

* La loi tchécoslovaque interdit la conduite des véhicules automobiles au consommateur d'une quantité quelconque d'alcool.

TR p. 423: «je proposai de la vodka; il refusa en faisant remarquer qu’il conduisait et que la loi interdit toute consommation d'alcool aux chauffeurs. ${ }^{6}$

Voici encore un autre exemple de la volonté d'éliminer systématiquement le paratexte explicatif: alors que M. Aymonin explique par deux notes qui sont Myslbek et Ales (TCH p. 90, TA p.101, TR p.137), M. Kundera semble considérer que le contexte de la phrase dans le texte tchèque de départ, permet de comprendre l'essentiel, c'est-à-dire qu'il s'agit d'artistes tchèques.

L'exemple qui suit, plus complexe, mêle adaptation et explication :

TCH p. 271: «Nevěřil bys, ale u prijímacích zkoušek na vysokou školu ti mladí už ani nevědí, co to byly procesy, Stalin je pro ně jen jméno, a Bucharin, Kameněv, Rajk už pro ně nejsou ani jména. Představ si, že vétšina z nich nevěděla ani, kdo byl Clementis. »

TA p. 300: «Tu ne le croiras pas, mais aux examens d'entrée en Faculté, ces jeunes-là ne savent même plus ce que c'était que les procès. Staline n'est qu'un nom pour eux; Boukharine, Kamenev, Rajk, pas même ça. Rends-toi compte que la plupart d'ente eux ne savaient pas qui était Clémentis !*»

*Intellectuel communiste slovaque, secrétaire d'Etat, puis Ministre des Affaires étrangères, condamné et exécuté, sous l'inculpation de "nationalisme bourgeois», lors des premiers grands procès de 1952 (en même temps que Rudolph Slansky et d'autres "traitres»), réhabilité à titre posthume.

TR p. 398: «Tu ne le croiras pas, mais aux examens d'entrée en Faculté, ces jeunes-là ne savent même plus ce que c'étati que les procès de Moscou, Staline n'est qu'un nom pour eux. Rends-toi compte que la plupart d'entre eux ne savent même pas qu'il y a dix ans ont eu lieu les procès politiques à Prague. »

M. Kundera ne gomme pas complètement ici la spécificité tchèque, mais il fait preuve aussi d'un souci d'adaptation. Tout d'abord, il emploie la façon française de désigner les procès en question. En effet l'expression «les procès de Moscou » correspond à la façon d'enseigner l'histoire en France, et il est certain que cette expression est plus parlante pour les Français que le simple mot "procès ». Ensuite il effectue une neutralisation en supprimant les noms propres des personnes passées en jugement et remplace le tout par une périphrase explicative à

\footnotetext{
${ }^{6}$ Un locuteur tchèque préciserait-il la loi tchèque sur la consommation d'alcool à un autre locuteur également tchèque ? Cette explication mise dans la bouche d'un des personnages du roman, semble s'adresser directement au lecteur français.
} 
peu près équivalente à la note de bas de page de M. Aymonin, « il y a dix ans ont eu lieu les procès politiques à Prague».

\section{Conclusion}

Les révisions de M. Kundera vont donc le plus souvent dans le sens d'une neutralisation des spécificités cognitivo-référentielles tchèques. C'est-à-dire qu'au contraire de M. Aymonin, qui a fait une traduction en "verres colorés », pour reprendre les termes de G.Mounin dans Les Belles infidèles, jouant de l'effet couleur locale -il a par exemple gardé les signes diacritiques de la langue tchèque - M. Kundera a cherché bien souvent une traduction en « verres transparents ». On a vu par ailleurs, que les quelques traits de la culture tchèque qui étaient conservés, étaient la plupart du temps expliqués au lecteur.

Quant à l'interprétation de ce choix de traduction, on peut faire plusieurs hypothèses. La première est très idéaliste : M. Kundera aurait cherché à s'adapter au lecteur français par souci d'universalité, faisant en sorte que son œuvre touche aussi des lecteurs ayant une autre expérience culturelle, tout écrivain espérant que son œuvre, partant du particulier, atteindra ce qu'il y a d'universel dans l'être humain. La deuxième hypothèse est beaucoup plus pragmatique : ce choix de traduction pourrait avoir aussi des motivations commerciales. Faire tomber la barrière culturelle permettrait de faciliter la lecture et donc une diffusion à un plus large public. Enfin il est également possible d'imaginer des motivations psychologiques, la difficulté pour M. Kundera de vivre entre deux cultures, la culture tchèque et la culture française, pouvant se résoudre par la volonté d'éliminer les éléments spécifiquement tchèques dans la traduction.

S'il lui importait peu de montrer dans les détails l'ancrage de son roman dans la culture tchèque, il était en revanche primordial pour $M$. Kundera que soit respecté dans son œuvre tout ce qui était de l'ordre du choix esthétique. Il explique d'ailleurs à ce sujet dans son essai Les Testaments trahis que ce sont les solutions esthétiques personnelles d'un romancier, qui permettent de dire qu'il participe à l'histoire du roman européen. Cela expliquerait notamment pourquoi M. Kundera a refusé l'adaptation que $M$. Aymonin a fait subir à la disposition du roman. En modifiant la présentation du texte tchèque, se composant de sept parties, chacune annoncée par le nom du personnage autour duquel elle est centrée, il semble que M. Aymonin soit passé à côté d'un des traits de l'originalité esthétique de M. Kundera. En effet la disposition du texte tchèque est à mettre en relation avec le travail du romancier sur les personnages, qui consiste en particulier à cerner le leitmotiv de leur existence.

\section{Bibliographie}

KUNDERA Milan (1967), Žert (exemplaire utilisé : Brno, Atlantis, 1996).

KunderA Milan (1968), La Plaisanterie, Paris, Gallimard, traduction de Marcel Aymonin. Kundera Milan (1985), La Plaisanterie, Paris, Gallimard, collection Folio, traduction révisée par Claude Courtot et l'auteur.

MounIN Georges (1955), Les Belles infidèles, Cahiers du Sud.

MoUNIN Georges (1963), Les problèmes théoriques de la traduction, Paris, Gallimard. 
SRPOVA Milena (1988-89), Problèmes de la traduction, enjeu d'une œuvre littéraire ?, Études tchèques et slovaques, Presses universitaires de Paris Sorbonne, p. 81-110.

SRPOVA Milena. (1995), La traduction, confrontation de deux expériences cognitives, Intellectica, 1, 20, p. 157-170. 


\section{ABSTRACT}

The process of translation does not only involve going from one language to another, but also from one reality to another, from one extra-linguistic world to another. The comparison between the first translation in French by Marcel Aymonin in 1968 of The Joke by Milan Kundera and the second translation, revised by the author and Claude Courtot in 1985, reveals the choices that every translator must do concerning the extralinguistic differences he faces. Several examples, such as the way Kundera translated proper names or how he dealt with the details of Czech history, enable us to say, that most of the time, Kundera tried to neutralize the Czech specificities while Aymonin kept them. 